\title{
Location Context Ontology Model based on Ubiquitous Computing Environment
}

\author{
Khamla Non Alinsavath ${ }^{1+}$, Lukito Edi Nugroho ${ }^{2}$, Widyawan $^{3}$ and Kazuhiko HAMAMOTO \\ ${ }^{123}$ Department of Electrical Engineering and Information Technology, Faculty of Engineering \\ Universitas Gadjah Mada, Yogyakarta, 55281, Indonesia \\ ${ }^{14}$ Department of Information Media Technology \\ School of Information and Telecommunication Engineering \\ Tokai University (Takanawa campus), Tokyo, Japan
}

\begin{abstract}
Many technologies have been used to identify the structure of context-based information in location-based service for positioning performance. Ontology based modeling is one of techniques that offer a domain that classify the type of location context entities. In order to represent the knowledge information in term of localization approach based indoor and outdoor conditions. This research proposes a location context ontology modeling to assort different locations that are linked together with multi-level of both indoor and outdoor conditions. A model provides the detail of proposed location context model and context reasoning in ubiquitous computing environment to test how location ontology can function at the same time and process the ability to infer more relations between location context entities.
\end{abstract}

Keywords: Ontology Model, Location Model, Location Awareness, Ubiquitous Computing.

\section{Introduction}

Positioning and localization technologies are integrating of sensing, processing, smart devices and human interaction to enhance high performance of information awareness. The advancement of these technologies performs importantly in numerous fields such as transportation, tourism and educational purposes. Location based service (LBS) [1] [2] uses the current position of mobile devices obtained from various technologies for the purpose of tagging, tracking, locating and monitoring. There are many technologies that available for localization, but the most famous of outdoor condition is the Global Positioning System (GPS) [3] and multiple technologies including Wi-Fi [4], Bluetooth and RFID (Radio Frequency Identification) [5] [6] that are used for indoor localization. By working under the complexity of physical and logical components, the system needs to find some schemes to deal with its' issues and uses for data representation. Ontology [7] [8] is a tool to represent the knowledge of localization, it is also an essential technique for the naming and description of different types, properties and interrelationship between different entities.

Ontologies open up a new area to deal with context-aware services. Therefore, the use of ontologies not only allows context modeling, but also to reason based on an inference engine and the collected data. Ontology is important model to share and exchange information between applications to the same domain, the conceptual of the knowledge representation includes the classes and their relationships of location-based service to demonstrate the entities to the model modes and apply to any condition of different environment. Ontology architecture assists user and developer to understand what concepts are present, which concepts are

\footnotetext{
+ Corresponding author. Tel.: + 6281226831422.

E-mail address: khamla@mail.ugm.ac.id.
} 
fundamental for location-based service and applications to categorize location entities in the right level of the ontology representing.

This research paper proposes the location context ontology model based on location based services (LBS). The model is designed by Hozo ontology editor software to identify different locations linked together with multi-level of both outdoor and indoor conditions. Specific details on the proposed location context model and context reasoning in ubiquitous computing environment are introduced. These include location representation, knowledge sharing and context ontology entities. This model explains the different components of large-scale location context until a specific positioning point is obtained to provide the direction and integration on the localization of outdoor and indoor seamlessly.

The remaining parts of the paper are divided into four sections. In section 2 presents the background and related work of ontology and location context knowledge. Section 3 identifies the location context ontology modeling. Section 4 describes the ontology location based service implementation. Finally, we summarize and outline the whole work at the section 5 .

\section{Background and Related Works}

Context [9] is any information that used to characterize the situation of an entity whereas an entity refers to place, object and people that are considered to be relevant to the interaction between user and an application such as location, time, activities and the preference of every entity. Context plays a significant role on the awareness information. These include a sensor context, a user context and an environment context. The operation of user context is recorded by the system for the reasoning and decision making of user's behavior and demands [10]. Context awareness means that one is able to use context information. It has the ability of a program or computing device to detect, sense, interpret, act and respond to different aspects of the environment such as location, time and user identity [11]. Context awareness system consists of many components such as context acquisition and sensing, context modeling and representation, context filtering and fusion, context storage and retrieval, and context application. This system uses context modeling and representation in the context awareness computing. Context modeling can be divided into two levels: different context use the data structure to express mode, such as K-Value models, markup scheme models. It can support the semantic unity such as ontology based models, graphical models, etc.

Context ontology [12] is a more uniformed language that can be used to develop a more complex universal systems. Throughout the process, context ontology demands very little effort to create a uniform model through its interaction with various context-aware systems. However, it is very difficult process to solemnize contextual information because of the presence of new information in the context aware computing system.

Several contexts are common and fundamental such as user, location, computational entity and activity for current situation. Therefore, ontology is used to describe concepts, attribute and relationship between related components in specific domain [13]. The ontology knowledge base is mainly used for storing ontology, context information, user model and reasoning rules.

Ontology based model [14] can be used to develop application that are capable of inferring context information that are relevant for the user. These models can be developed using web ontology language (OWL) [15] that has the structure of a dynamic database, which is efficient for location-based service searching development. Ontology is conceived as a set of entities, relations, functions, axioms and instances. For developing context models based on ontology [16] regards to several reasons as following:

- Knowledge sharing: the use of context ontology enables computational entities such as agents and services in pervasive computing environments to have a common set of concept about context while interaction with one another.

- Logic reference: based on ontology, context aware computing can exploit various existing logic reasoning mechanism to deduce high-level. Conceptual context from low-level, raw context, and to check and solve inconsistent context knowledge due to imperfect sensing.

- Knowledge reuse: by using well-defined web ontologies of different domains. These include the temporal and spatial ontology. 
In [17] proposed a location-based service using ontology approach with a focus on the indoor activities in a university context. The model was designed for sharing, managing and querying data semantically, reasoning rules were created for semantic queries to retrieve and provide information. The framework was proposed to describe the entire location-based service system and its components; a 3D network model-based topological data model was used to calculate the shortest path to a destination location of interest. This study contributed to further implementation of ontology reasoning techniques that were integrated with locationbased, the reasoning rules also enhances the richness of semantic queries for suggesting other places for more choice, relevant to destination of interest.

In [18] presented the model of the navigation of the ILONA system to model the structure of building efficiently and this model can be used to extend the ILONA system with an ontology-based navigation capability. The extended model allowed the grouping of zones based on their physical location, owner or function that made the handing of zones easier and enhances the modeling ability of the ILONA system. The navigation components also defines gateway between zones that will be used by the routing algorithms. Automatic tests more than 90 percent of the source code of the component presented.

In [19] proposed a medical assistance system based on ontologies that manage and exploit the larger and rapidly growing volume of medical data in order to facilitate on the road decision making for the mobile physician. Ontology-based medical system allows finding out as quickly as possible the needed medical resources and reserves the most suitable health care institutions according to the patient state. The information provided a great support to match patients and physicians needs with medical resources and skilled staffs available in the nearest health care institutions.

\section{Location Context Ontology Modeling}

Many existing models have been developed by researchers. These include the symbolic models, hierarchical models, and geometric models. In this research paper, the model-based ontology integrates with symbolic models to support the system based outdoor and indoor scenarios seamlessly. The models are supported to be the framework for the whole conceptual of the proposed system. The models are expected to provide both outdoor and indoor localization that enable users to have a greater awareness of the scope context location from larger scope until specific positioning is achieved. The system integrates indooroutdoor seamlessly and does not take into consideration on the boundary between global and local positioning.

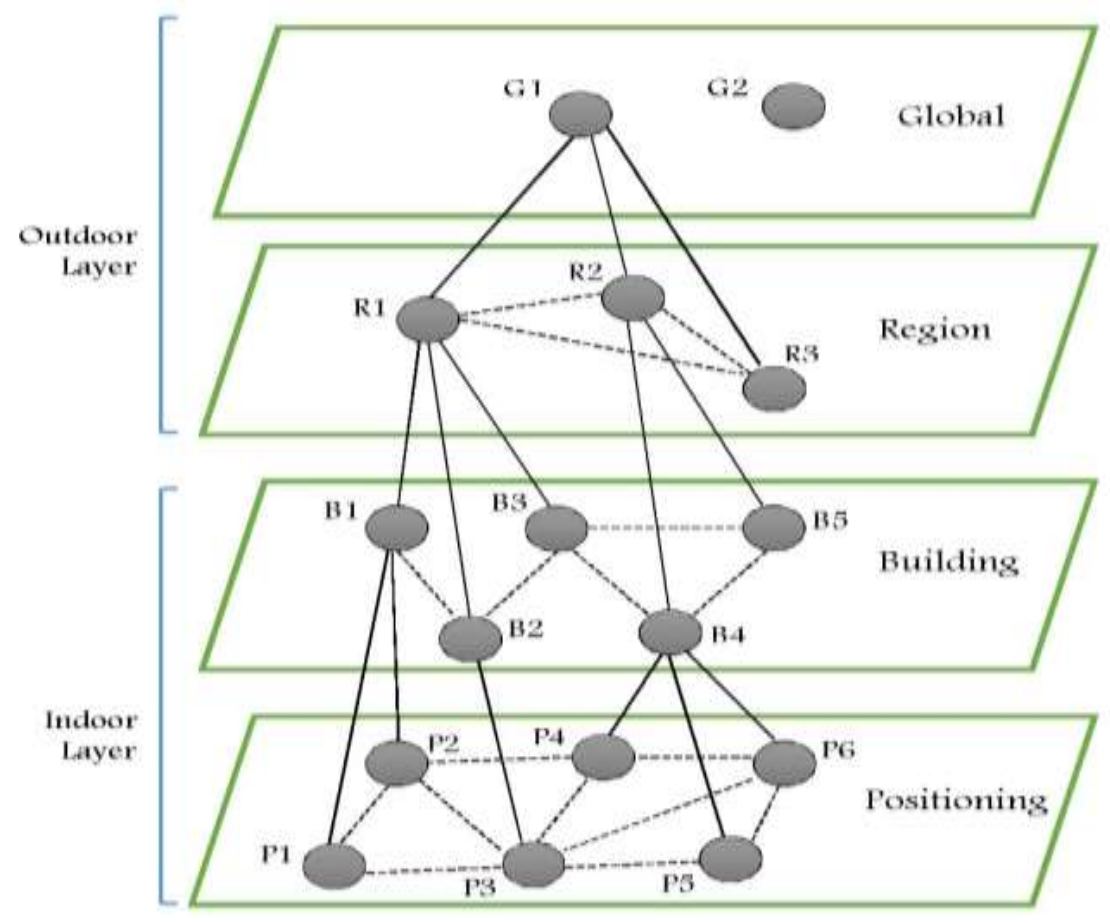

Fig. 1: Symbolic model based large scale of location specification. 
The high level scenario of the proposed models can be represented by any attribute such as zone, continent, country and so on, that give a better primary conceptual framework of the system. Moreover the lower levels of information can expand when zoomed in from the higher levels of information based on user's current location. This information not only provides the user location, but also enables the system to supply user information such as people nearby, user profile and point of interests. The proposed frameworks illustrate the relationship between higher level and lower level seamlessly. As such, the framework supports both outdoor and indoor technologies after the system has adjusted to specific technologies according to the current situation. The route can then be provided to reach specified user's target direction.

Symbolic location model (Figure 1) utilizes the positioning from a large scale to specific space. As a result, it can be used to express all physical entities with whole properties and objects. A model provides a different perspective at expressing related components and the relationship between symbolic model and the environment. The proposed model was defined relatively to the global of discourse such as geo-location scenarios, tourism guidance, metropolitan planning. As the result, the structure model has been designed to be more users friendly by providing support to the ubiquitous environment.

The designed model location context awareness is common for both geo-location and relevant physical objects concepts. A location is explained by geographical perspectives, positioning tracking and taxonomy for location determination. Physical objects are component related to location awareness and navigation service such as smart devices, activity, process and technology. In several accessions, time ontology is used to fulfill the requirement of real-time responding service for the real situation. The system can then be used to determine location everywhere from the proposed system.

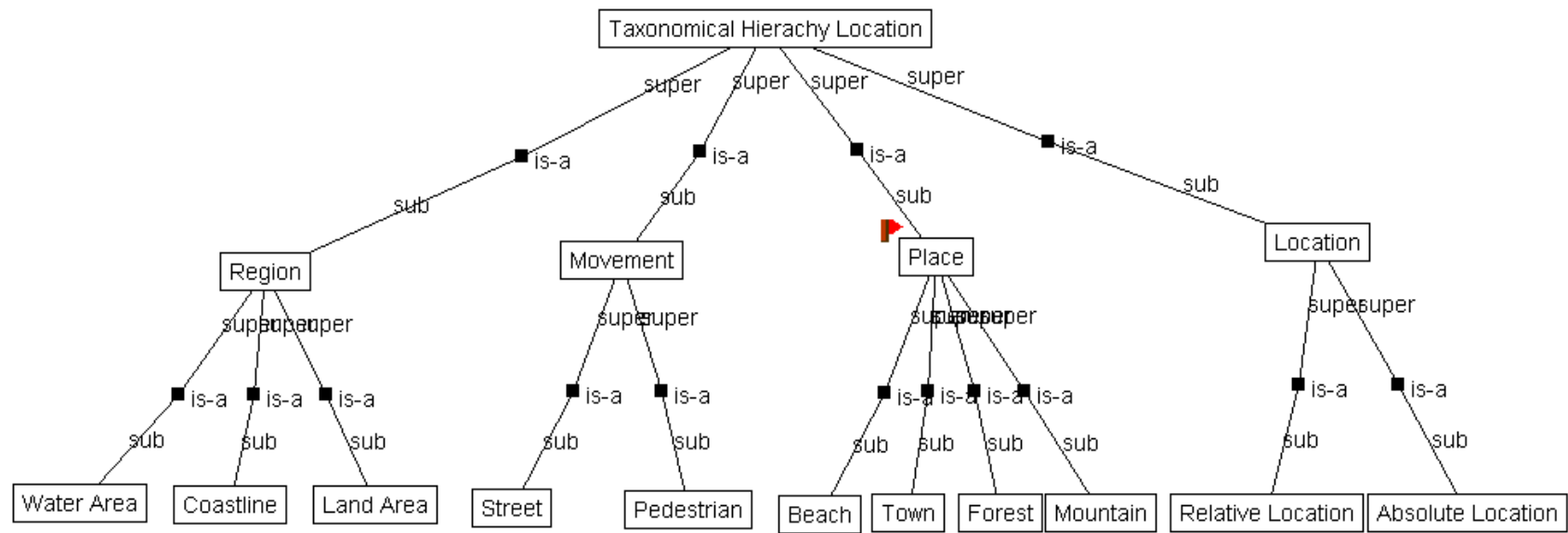

Fig. 2: The general classes of the location taxonomy.

The taxonomical hierarchy location (Figure 2) has been proposed to represent the general term of location entities that enable to provide to any particular location. The components of location entities such as region, movement, place and location are defined as the geographical ontology structure to identify conceptual among knowledge exchange from the large scale to the specified positioning. The various relations to formulize the physical objects in term of appropriate modeling that can be investigated the different class of the object properties. The location information to represent each sub entities are described the integration between geo-information. The root of the graph is defined as taxonomical hierarchy location to represent any location in the world. The classes of \{region, movement, place and location\} are divided into sub-classes such as region class is divided into \{water area, coastline and land area\}. Movement can be divided into \{street and pedestrian\}. Place \{beach, town forest and mountain\}, Location \{Absolute location and relative location\}. For other sub-classes can be divided further extraction granularity which depend on the provided geographical region and appropriate location context.

The infrastructure of the localization (Figure 3) supports the context awareness computing service in term of pervasiveness and ubiquity environment which is consisted of evaluation properties and context information such as current location, current time. It contains a relation location layers and context that serve and provide the service to the user accurately. Hence, the specification of these approaches enable the 
support of the right service irrespective the user situation, environment changes. Indoor condition that shown in the Figure 3 can be accommodated to all possibilities in the context domain in terms of indoor entities representation, however, this graph explained the entities of university context as an example for the proposed system. Sub-entities of the indoor location such as footpath, lobby, floor and room are able to apply to the other perspectives based indoor condition such as concert hall, indoor station and shopping center.

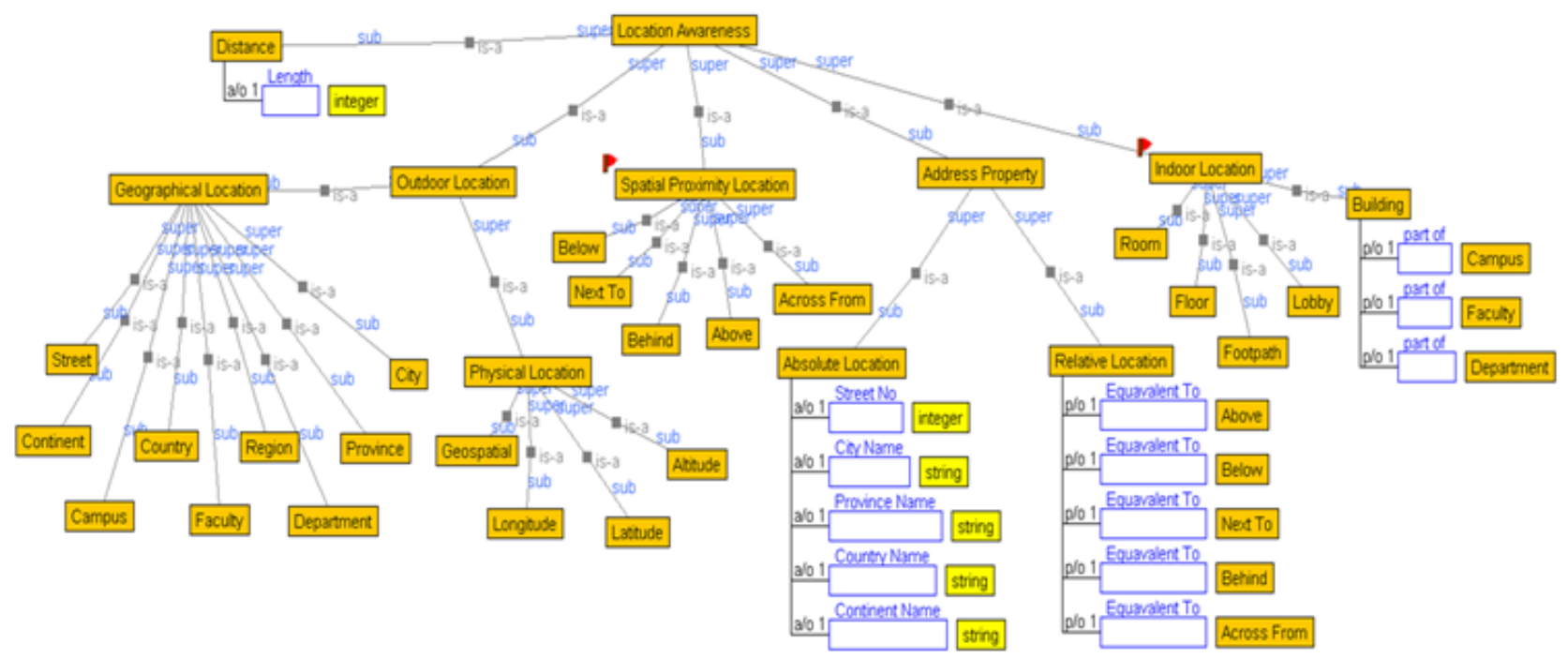

Fig. 3: Location Context Awareness Hierarchy graph.

Within the navigation service scenario, the activities include travelling, moving, guidance and working. These activities also contain sub-components. On the other hand, the service includes many types of relevant knowledge representations namely user, technology, device, environment that are listed in the Figure 4 (left). Based on Figure 4 (left), the type and elements of the service play an important role by identifying the correct factor that specifies on the exact positioning in the location context based indoor and outdoor conditions.
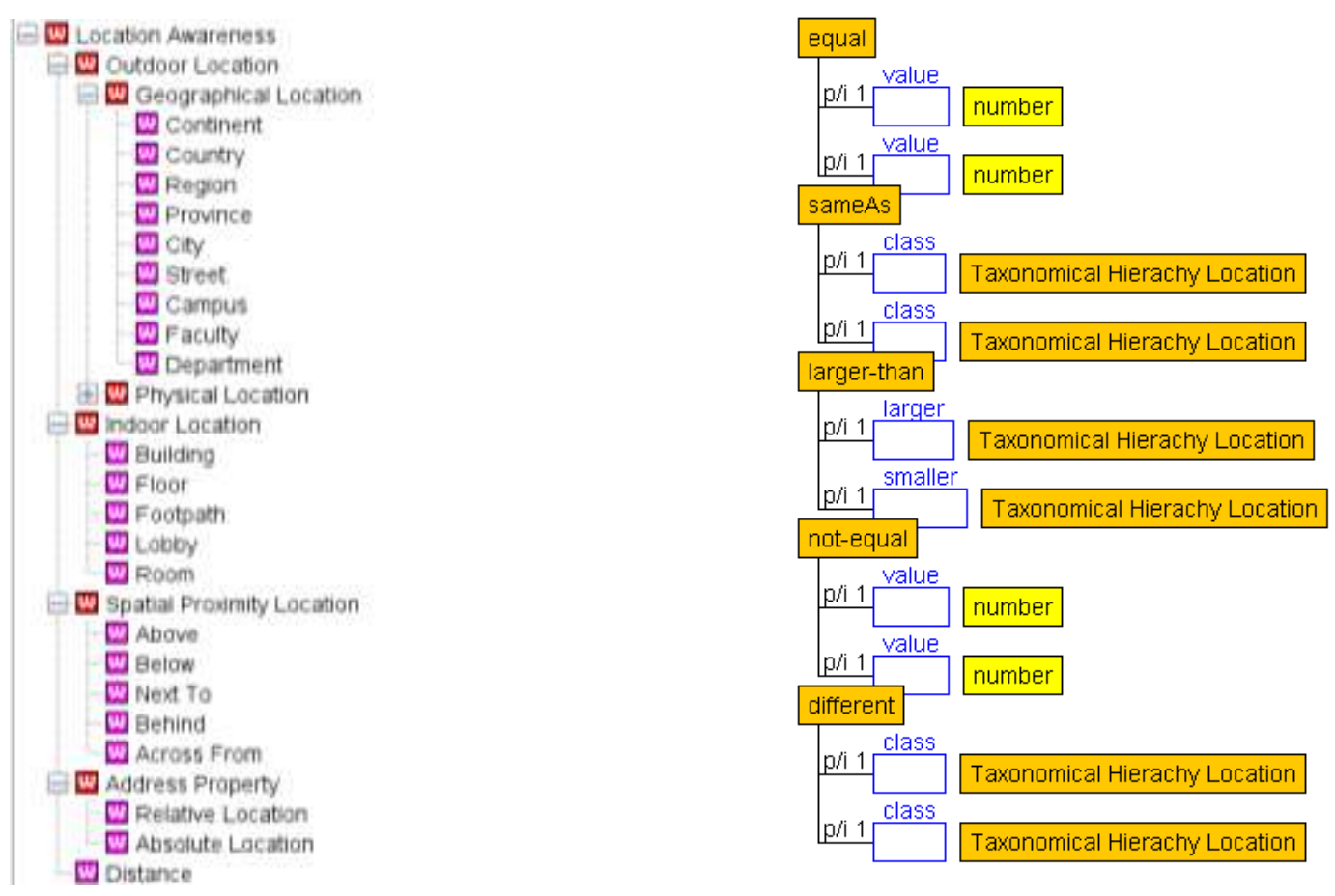

Fig. 4: Location Context Entities (Left) and Relation concept of the taxonomy location (Right).

\section{Ontology Location Based Service Implementation}

Location context ontology models have been developed to provide the information on the geographical location and other components that are related to location awareness approaches, this category has been 
analyzed and the structure of model has been created based on the formation of the logical analysis to infer additional relationship between location context entities. The model has been developed by Hozo ontology editor software version 5.6.2 bata [20] that is built on the ontology web language to support and classify the different classes and object properties of the location based indoor and outdoor conditions. Location ontologies are mapped to the root class called "Navigation Service" and disjoint classes that are connected to one another.

Location context entities and individual by class (Figure 4) are used to characterize any geographical location such as absolute location and relative location, the meaning of each entities and their relation with another related entities are also used to represent the knowledge information in the hierarchy taxonomical concepts, any deployment areas that can be classified by their location scales such as continent, region, country, province and city. Furthermore physical location and spatial proximity location have been identified using value of positioning coordinates such as \{longitude, latitude, above, across, behind, below and etc.\}.

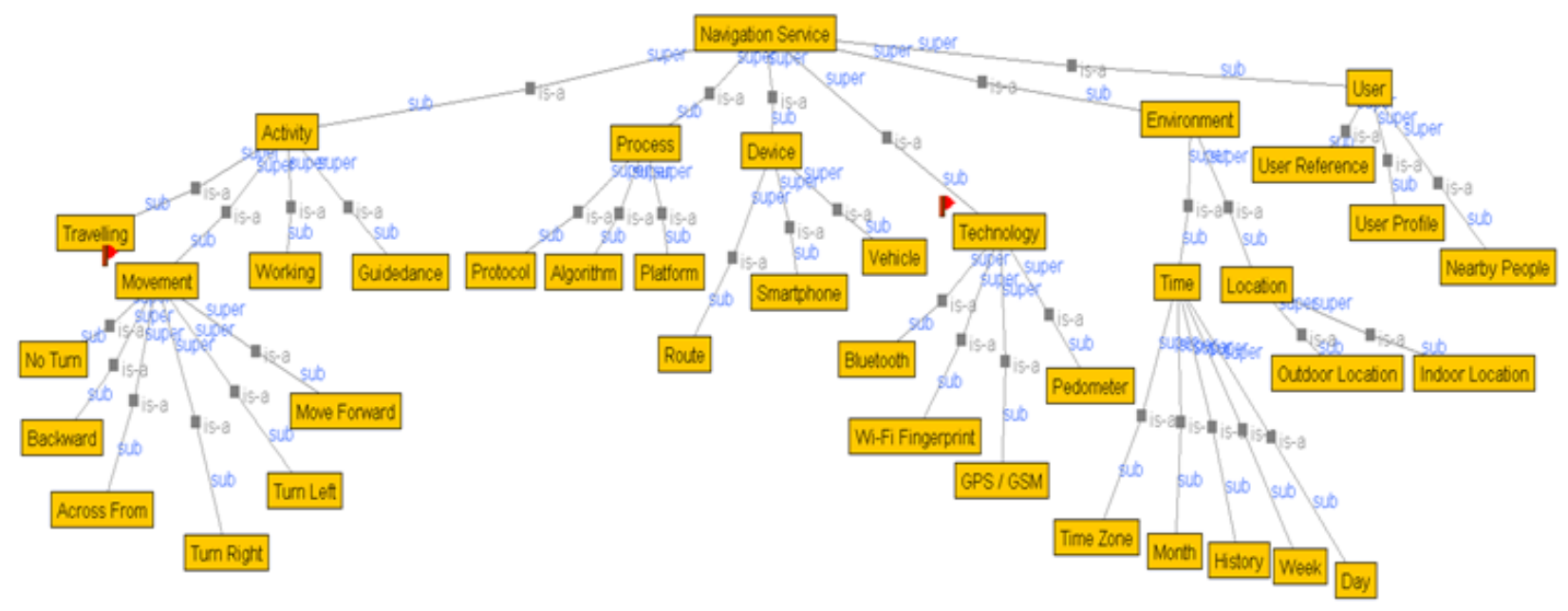

Fig. 5: Navigation Service scenarios elements.

We have added classes and important concepts such as subclasses of geographical location based indoor and outdoor conditions. The overlapped areas are classified into two parts, namely the outdoor local and indoor global (Figure 5) to display specific positioning entities for the separation of subsections at the boundary of indoor and outdoor locations. The concept of outdoor local has been provided in terms of university ontology context including \{campus, faculty, department and building\}; in regards to indoor global, information include \{footpath, floor, and room $\}$.

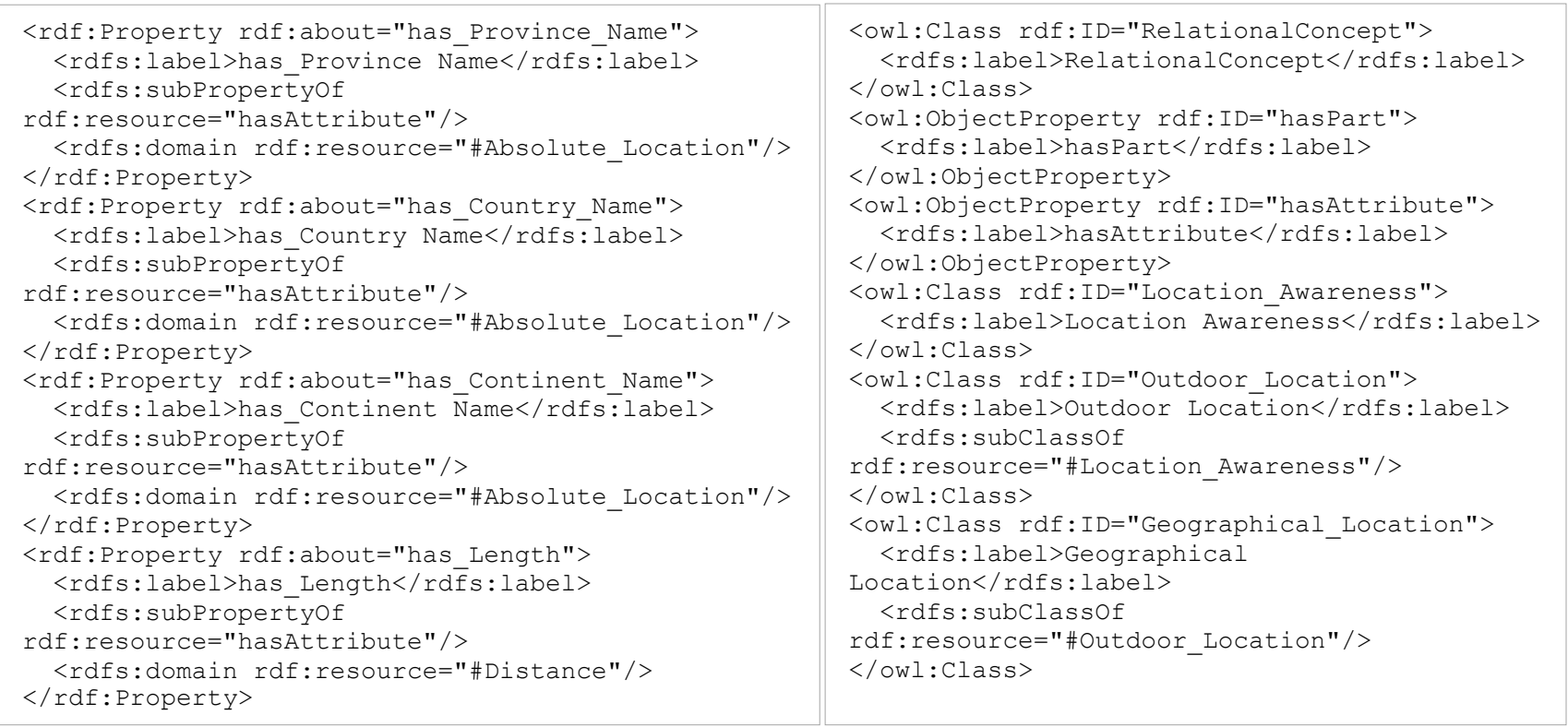

Fig. 6: Resource Description Framework of localization (Left) and Ontology Web Language output (Right) 
In addition, the overall process of the ontology framework comprises a set of relation between data properties and classes (object properties). The object property specifies the relationship between outdoor and indoor location such as continent, country and to find continent that contains the searched country and provinces that are part of country, and cities that are contained in the province properties.

Table 1: Set of object properties for location ontology

\begin{tabular}{|l|l|l|l|l|}
\hline \multicolumn{1}{|c|}{ Entities } & \multicolumn{1}{c|}{ Object Properties } & \multicolumn{1}{c|}{ Data Properties } & \multicolumn{1}{c|}{ Individual by classes } & \multicolumn{1}{c|}{ Range } \\
\hline Continent & Include & Continent_name & Asia & String \\
\hline Country & Involve & Country_name & Japan & String \\
\hline Province & Is_part_of & Province_name & Tokyo & String \\
\hline City & Is_part_of & City_name & Shibuya-ku & String \\
\hline
\end{tabular}

In order to facilitate decision-making, the system needs to query ontology of localization entities. It contains property path expressions that allow path properties to be matched, the purposes of query analysis and query plan optimization are provided to implement the query based on query execution. The query supports the keywords, meaning to become solution and can be subtracted from another provided form to fulfill the complex class expressions and essentially query ontology entities.

\section{Conclusion}

This research paper introduces an ontology-based model to support ubiquitous computing environment based location awareness application. The model is built to be developed as a framework for the localization based indoor and outdoor conditions and to specify the different component of navigation service. In addition, the paper provides a comprehensive explanation on the sub-elements of the ontology-based model. We define different types of localizations and related components of the location-based service approach.

The proposed ontology model using Hozo ontology editor software to develop the prototype model can be used in any location-based service application to easily realize the domain, concepts and its relations, any other tool or language except Hozo ontology editor can also be used to indicate the entire location entities for understandable representation of ontologies, on the other hand, the ontology can provide in translating the location-based data such as geographical location into semantic information.

The implementation of the ontology relationship is also provided in detail in terms of location taxonomy. Having said this, this model is adaptable to any individual depending on their fields. Furthermore, developers can utilize the information in the paper to support and to guide their work with other knowledge and for further improvement. This model is a beginning procedure of the large project of the integration between different condition of localization and being a model to develop localization awareness application. Even though challenges remain on the exploration and the implementation of the model due to real-time responding and higher performance based location awareness approach, the model can be used as a starter kit to develop localization awareness application.

\section{Acknowledgements}

I would like to express my deep acknowledgement to those who have assisted, supported and encouraged me while carrying out this research. This includes my supervisors and colleagues, as well as the financial support from the AUN/SEED-Net JICA Project and Tokai University.

\section{References}

[1] A. Zipf and M. M. Jöst, "Location-Based Services," in Springer Handbook of Geographic Information, W. Kresse and D. M. Danko, Eds. Berlin, Heidelberg: Springer Berlin Heidelberg, 2012, pp. 417-421.

[2] L. Hu, Q. Tong, Z. Du, Y. Liu, and Y. Tang, "Location-based service using ontology and collaborative recommendation," in 2014 International Conference on Information Science, Electronics and Electrical Engineering, 2014, vol. 1, pp. 653-656.

[3] S.-C. Yeh, W.-H. Hsu, M.-Y. Su, C.-H. Chen, and K.-H. Liu, “A study on outdoor positioning technology using GPS and WiFi networks," in 2009 International Conference on Networking, Sensing and Control, 2009, pp. 597601 . 
[4] E. Jedari, Z. Wu, R. Rashidzadeh, and M. Saif, "Wi-Fi based indoor location positioning employing random forest classifier," in 2015 International Conference on Indoor Positioning and Indoor Navigation (IPIN), 2015, pp. 1-5.

[5] M. J. Deka, J. Joshi, N. Sinha, A. Tyagi, A. Kushal, and A. Jain, "Indoor and outdoor position identification using RFID," in 2016 International Conference on Recent Advances and Innovations in Engineering (ICRAIE), 2016, pp. 1-7.

[6] K. Mannay, N. Benhadjyoussef, M. Machhout, and J. Ureña, "Location and Positioning Systems: Performance and comparison," in 2016 4th International Conference on Control Engineering Information Technology (CEIT), 2016, pp. 1-6.

[7] J. R. Hilera and F. Ruiz, "Ontologies in Ubiquitous Computing.," in ICUC, 2006.

[8] H. Chen, T. Finin, and A. Joshi, "An Ontology for Context-aware Pervasive Computing Environments," Knowl Eng Rev, vol. 18, no. 3, pp. 197-207, Sep. 2003.

[9] J. Hong, E. Suh, and S.-J. Kim, “Context-aware systems: A literature review and classification,” Expert Syst. Appl., vol. 36, pp. 8509-8522, 2009.

[10] Z. Ming and M. Yan, "Study on the ontology-base context-aware and reasoning model of IOT," in IEEE Conference Anthology, 2013, pp. 1-5.

[11] M. Baldauf, S. Dustdar, and F. Rosenberg, "A survey on context-aware systems," Int. J. Ad Hoc Ubiquitous Comput., vol. 2, no. 4, pp. 263-277, 2007.

[12] S. Malik and S. Jain, "Ontology based context aware model," in 2017 International Conference on Computational Intelligence in Data Science(ICCIDS), 2017, pp. 1-6.

[13] S. Alhazbi, L. Lotfi, R. Ali, and R. Suwailih, "Ontology-based model in tourism context-aware systems," in 2013 International Conference on ICT Convergence (ICTC), 2013, pp. 775-779.

[14] A. H. O. R. Castillo, F. N. Buzeto, C. D. Castanho, and R. P. Jacobi, “An Ontology-Based Model for Context Information Management in Smart Spaces," in 2013 IEEE 10th International Conference on Ubiquitous Intelligence and Computing and 2013 IEEE 10th International Conference on Autonomic and Trusted Computing, 2013, pp. 278-284.

[15] W. Chaochaisit, M. Bessho, N. Koshizuka, and K. Sakamura, "Human Localization Sensor Ontology: Enabling OWL 2 DL-Based Search for User's Location-Aware Sensors in the IoT," in 2016 IEEE Tenth International Conference on Semantic Computing (ICSC), 2016, pp. 107-111.

[16] X. H. Wang, D. Q. Zhang, T. Gu, and H. K. Pung, "Ontology based context modeling and reasoning using OWL," in IEEE Annual Conference on Pervasive Computing and Communications Workshops, 2004. Proceedings of the Second, 2004, pp. 18-22.

[17] K. Lee, J. Lee, and M.-P. Kwan, "Location-based service using ontology-based semantic queries: A study with a focus on indoor activities in a university context," Comput. Environ. Urban Syst., vol. 62, pp. 41-52, 2017.

[18] D. P. Kun, E. B. Varga, and Z. Toth, "Ontology based navigation model of the ILONA system," in 2017 IEEE 15th International Symposium on Applied Machine Intelligence and Informatics (SAMI), 2017, pp. 000479000484.

[19] L. Mhadhb, J. Akaichi, M. H. Karray, and B. Archimde, "Ontologies for location based services quality enhancement: the case of emergency services," in 2015 Second International Conference on eDemocracy eGovernment (ICEDEG), 2015, pp. 90-97.

[20] “Hozo - Ontology Editor.” [Online]. Available: http://www.hozo.jp/download_en.php. [Accessed: 20-Feb-2019]. 